\title{
Piecewise continuous approach to nonlinear differential equations approximation problem of computational structural mechanics
}

Roman Leibov ${ }^{*}$

Moscow State University of Civil Engineering, Yaroslavskoe shosse, 26, Moscow, 129337, Russia

\begin{abstract}
This paper presents a nonlinear differential equations system piecewise continuous approximation. The piecewise continuous approximation improves piecewise linear approximation through reducing the errors at the boundaries of different linear differential equations systems areas. The matrices of piecewise continuous differential and algebraic equations systems are estimated using nonlinear differential equations system time responses and random search method. The results of proposed approach application are presented.
\end{abstract}

\section{Piecewise linear and piecewise continuous approximations of nonlinear differential equations system}

Let's consider nonlinear differential equations system [1-4].

$$
\dot{\mathbf{x}}=\mathbf{f}(\mathbf{x}, \mathbf{u}) \text {. }
$$

There are $\mathbf{x}$ - vector of state variables, $\mathbf{u}$ - vector of input variables.

The vector of constant input variables ${ }_{s} \mathbf{u}$ determines the vector of steady-state state variables ${ }_{\mathrm{s}} \mathbf{x}$.

Nonlinear differential equations system is approximately described by linear differential equations system in a small neighborhood of $r$-th steady-state point [1-4].

$$
\dot{\mathbf{x}}^{\mathrm{n}}={ }^{r} \mathbf{A}\left(\mathbf{x}^{\mathrm{n}}-{ }_{\mathrm{s}}^{r} \mathbf{x}^{\mathrm{n}}\right)+{ }^{r} \mathbf{B}\left(\mathbf{u}^{\mathrm{n}}-{ }_{\mathrm{s}}^{r} \mathbf{u}^{\mathrm{n}}\right) .
$$

For steady-state values of input and state variables deviations

$$
\mathbf{0}={ }^{r} \mathbf{A}\left(\mathbf{x}^{\mathrm{n}}-{ }_{\mathrm{S}}^{r} \mathbf{x}^{\mathrm{n}}\right)+{ }^{r} \mathbf{B}\left(\mathbf{u}^{\mathrm{n}}-{ }_{\mathrm{S}}^{r} \mathbf{u}^{\mathrm{n}}\right), \quad\left(\mathbf{x}^{\mathrm{n}}-{ }_{\mathrm{s}}^{r} \mathbf{x}^{\mathrm{n}}\right)=-{ }^{r} \mathbf{A}^{-1}{ }^{r} \mathbf{B}\left(\mathbf{u}^{\mathrm{n}}-{ }_{\mathrm{s}}^{r} \mathbf{u}^{\mathrm{n}}\right)={ }^{r} \mathbf{S}\left(\mathbf{u}^{\mathrm{n}}-{ }_{\mathrm{s}}^{r} \mathbf{u}^{\mathrm{n}}\right) .
$$

Therefore

$$
{ }^{r} \mathbf{B}=-{ }^{r} \mathbf{A}{ }^{r} \mathbf{S}
$$

There are $\mathbf{x}^{\mathrm{n}}$ - vector of normalized state variables, $\mathbf{u}^{\mathrm{n}}-$ vector of normalized input variables,

$$
x_{i}^{\mathrm{n}}=x_{i} / \max _{r=1, \ldots, R}\left|{ }_{\mathrm{s}}^{r} x_{i}\right|, \quad i=1, \ldots, n, \quad u_{i}^{\mathrm{n}}=u_{i} / \max _{r=1, \ldots, R}\left|\underset{\mathrm{s}}{r} u_{i}\right|, \quad i=1, \ldots, m,
$$

\footnotetext{
* Corresponding author: r_leibov@mtu-net.ru
} 
${ }^{r} \mathbf{A},{ }^{r} \mathbf{B},{ }^{r} \mathbf{S}$ are linear differential and linear algebraic equations systems matrices for $r$-th steady-state point,

${ }_{s}^{r} \mathbf{u}^{\mathrm{n}},{ }_{\mathrm{s}}^{r} \mathbf{x}^{\mathrm{n}}$ are vectors of normalized input and state variables for $r$-th steady-state point,

${ }^{r} \mathbf{A},{ }^{r} \mathbf{B},{ }^{r} \mathbf{S},{ }_{\mathrm{s}}^{r} \mathbf{u}^{\mathrm{n}},{ }_{\mathrm{s}}^{r} \mathbf{x}^{\mathrm{n}}, r=1,2, \ldots, R$ are piecewise linear differential equations system matrices, piecewise linear algebraic equations system matrices and vectors of normalized input and state variables for $R$ neighboring steady-state points.

Let's define the vectors of normalized input and state variables and piecewise continuous linear differential and linear algebraic equations systems matrices as follows

$$
\begin{aligned}
& { }_{\mathrm{s}} \mathbf{u}^{\mathrm{n}}={ }_{\mathrm{s}}^{r} \mathbf{u}^{\mathrm{n}}+\theta\left({ }_{\mathrm{s}}^{r+1} \mathbf{u}^{\mathrm{n}}-{ }_{\mathrm{s}}^{r} \mathbf{u}^{\mathrm{n}}\right), \quad{ }_{\mathrm{s}} \mathbf{x}^{\mathrm{n}}={ }_{\mathrm{s}}^{r} \mathbf{x}^{\mathrm{n}}+\theta\left({ }_{\mathrm{s}}^{r+1} \mathbf{x}^{\mathrm{n}}-{ }_{\mathrm{s}}^{r} \mathbf{x}^{\mathrm{n}}\right), \quad r=1, \ldots, R-1, \\
& \mathbf{A}={ }^{r} \mathbf{A}+\theta\left({ }^{r+1} \mathbf{A}-{ }^{r} \mathbf{A}\right), \quad \mathbf{S}={ }^{r} \mathbf{S}+\theta\left({ }^{r+1} \mathbf{S}-{ }^{r} \mathbf{S}\right), \quad \mathbf{B}=-\mathbf{A} \mathbf{S}, \quad r=1, \ldots, R-1, \\
& \theta=\left(\left\|\mathbf{x}^{\mathrm{n}}\right\|-\left\|{ }_{\mathrm{s}}^{r} \mathbf{x}^{\mathrm{n}}\right\|\right) /\left(\left\|{ }_{\mathrm{s}}^{r+1} \mathbf{x}^{\mathrm{n}}\right\|-\left\|{ }_{\mathrm{s}}^{r} \mathbf{x}^{\mathrm{n}}\right\|\right) \text {. }
\end{aligned}
$$

There are

$$
\begin{gathered}
r=1 \mid\left\|\mathbf{x}^{\mathrm{n}}\right\| /\left\|{ }_{\mathrm{s}}^{R} \mathbf{x}^{\mathrm{n}}\right\| \leq{ }_{\mathrm{s}}^{2} \mathbf{x}^{\mathrm{n}}\|/\|{ }_{\mathrm{s}}^{R} \mathbf{x}^{\mathrm{n}} \|, \\
r=2, \ldots, R-2 \mid\left\|{ }_{\mathrm{s}}^{r} \mathbf{x}^{\mathrm{n}}\right\| /\left\|{ }_{\mathrm{s}}^{R} \mathbf{x}^{\mathrm{n}}\right\|<\left\|\mathbf{x}^{\mathrm{n}}\right\| /\left\|{ }_{\mathrm{s}}^{R} \mathbf{x}^{\mathrm{n}}\right\| \leq\left\|{ }_{\mathrm{s}}^{r+1} \mathbf{x}^{\mathrm{n}}\right\| /\left\|{ }_{\mathrm{s}}^{R} \mathbf{x}^{\mathrm{n}}\right\|, \\
r=R-1\left\|{ }_{\mathrm{s}}^{R-1} \mathbf{x}^{\mathrm{n}}\right\| /\left\|{ }_{\mathrm{s}}^{R} \mathbf{x}^{\mathrm{n}}\right\|<\left\|\mathbf{x}^{\mathrm{n}}\right\| /\left\|{ }_{\mathrm{s}}^{R} \mathbf{x}^{\mathrm{n}}\right\| .
\end{gathered}
$$

\section{Piecewise continuous differential and linear algebraic equations system matrices estimation using nonlinear differential equations time responses}

Nonlinear differential equations time responses ${ }^{\mathrm{NL}} \mathbf{u}^{\mathrm{n}}\left(t_{k}\right), \quad k=0, \ldots, N-1,{ }^{\mathrm{NL}} \mathbf{x}^{\mathrm{n}}\left(t_{k}\right)$, $k=0, \ldots, N$, where $t_{k+1}=t_{k}+\Delta t, k=0, \ldots, N-1$, are used for piecewise continuous linear differential equations and linear algebraic equations systems matrices estimation. These matrices and known vectors of normalized input and state variables ${ }_{\mathrm{s}}^{r} \mathbf{u}^{\mathrm{n}},{ }_{\mathrm{s}}^{r} \mathbf{x}^{\mathrm{n}}, r=1,2, \ldots, R$ correspond to $R$ neighboring steady-state points. The ${ }^{\mathrm{NL}} \mathbf{u}^{\mathrm{n}}\left(t_{k}\right), k=0, \ldots, N-1$ represent several consecutive stepwise time responses which connect different ${ }_{\mathrm{s}}^{r} \mathbf{u}^{\mathrm{n}}, r=1,2, \ldots, R$.

Piecewise-continuous linear differential equations and linear algebraic equations systems matrices estimation can be reduced to nonlinear programming problem

$$
\begin{aligned}
& { }^{r} \mathbf{A},{ }^{r} \mathbf{S}, r=1,2, \ldots, R: \min \left\{\sqrt{\frac{\sum_{k=1}^{N}\left[\mathbf{x}^{\mathrm{n}}\left(t_{k}\right)-{ }^{\mathrm{HЛ}} \mathbf{x}^{\mathrm{n}}\left(t_{k}\right)\right]_{\mathbf{x}}^{T} \mathbf{W}\left(t_{k}\right)\left[\mathbf{x}^{\mathrm{n}}\left(t_{k}\right)-{ }^{\text {НЛ }} \mathbf{x}^{\mathrm{n}}\left(t_{k}\right)\right]}{\sum_{k=1}^{N}\left[{ }^{\mathrm{Hл}} \mathbf{x}^{\mathrm{n}}\left(t_{k}\right)-{ }^{\text {НЛ }} \mathbf{x}^{\mathrm{n}}\left(t_{0}\right)\right]^{T} \mathbf{x} \mathbf{W}\left(t_{k}\right)\left[{ }^{\mathrm{НЛ}} \mathbf{x}^{\mathrm{n}}\left(t_{k}\right)-{ }^{\mathrm{HЛ}} \mathbf{x}^{\mathrm{n}}\left(t_{0}\right)\right]}} \mid\right. \\
& \mathbf{x}^{\mathrm{n}}\left(t_{k+1}\right) \approx \mathbf{x}^{\mathrm{n}}\left(t_{k}\right)+\Delta t \mathbf{A}\left[\mathbf{x}^{\mathrm{n}}\left(t_{k}\right)-{ }_{\mathrm{s}} \mathbf{x}^{\mathrm{n}}\right]-\Delta t \mathbf{A} \mathbf{S}\left[{ }^{\text {нл }} \mathbf{u}^{\mathrm{n}}\left(t_{k}\right)-{ }_{\mathrm{s}} \mathbf{u}^{\mathrm{n}}\right], \\
& { }_{\mathrm{s}} \mathbf{u}^{\mathrm{n}}={ }_{\mathrm{s}}^{r} \mathbf{u}^{\mathrm{n}}+\theta\left({ }_{\mathrm{s}}^{r+1} \mathbf{u}^{\mathrm{n}}-{ }_{\mathrm{s}}^{r} \mathbf{u}^{\mathrm{n}}\right), \quad{ }_{\mathrm{s}} \mathbf{x}^{\mathrm{n}}={ }_{\mathrm{s}}^{r} \mathbf{x}^{\mathrm{n}}+\theta\left({ }_{\mathrm{s}}^{r+1} \mathbf{x}^{\mathrm{n}}-{ }_{\mathrm{s}}^{r} \mathbf{x}^{\mathrm{n}}\right), \quad r=1, \ldots, R-1, \\
& \mathbf{A}={ }^{r} \mathbf{A}+\theta\left({ }^{r+1} \mathbf{A}-{ }^{r} \mathbf{A}\right), \quad \mathbf{S}={ }^{r} \mathbf{S}+\theta\left({ }^{r+1} \mathbf{S}-{ }^{r} \mathbf{S}\right), \quad \mathbf{B}=-\mathbf{A} \mathbf{S}, \quad r=1, \ldots, R-1,
\end{aligned}
$$




$$
\left.\theta=\left(\left\|\mathbf{x}^{\mathrm{n}}\left(t_{k}\right)\right\|-\left\|{ }_{\mathrm{s}}^{r} \mathbf{x}^{\mathrm{n}}\right\|\right) /\left(\left\|{ }_{\mathrm{s}}^{r+1} \mathbf{x}^{\mathrm{n}}\right\|-\left\|{ }_{\mathrm{s}}^{r} \mathbf{x}^{\mathrm{n}}\right\|\right), \quad k=0,1, \ldots, N-1\right\} .
$$

There are ${ }_{\mathbf{x}} \mathbf{W}\left(t_{k}\right), k=1, \ldots, N$ positive definite diagonal weight matrices, $\mathbf{x}^{\mathrm{n}}\left(t_{0}\right)={ }^{\mathrm{HЛ}} \mathbf{x}^{\mathrm{n}}\left(t_{0}\right)$. The nonlinear programming problem is solved by the random search method. The initial values of piecewise-continuous differential and algebraic equations systems matrices are piecewise linear differential and algebraic equations systems matrices.

\section{Piecewise-continuous linear differential and linear algebraic equations systems matrices estimation example}

In this example $m=4, n=5$, the vectors of normalized steady-state values of input and state variables for $R=6$ are

$$
\begin{gathered}
{ }_{\mathrm{s}}^{6} \mathbf{u}^{\mathrm{n}}=[1,0.4515,0,0]^{T}, \\
{ }_{\mathrm{s}}^{6} \mathbf{x}^{\mathrm{n}}=[1,1,1,1,1]^{T}, \\
{ }_{\mathrm{s}}^{5} \mathbf{u}^{\mathrm{n}}=[0.9442,0.4441,-0.0893,0]^{T}, \\
{ }_{\mathrm{s}} \mathbf{x}^{\mathrm{n}}=[0.9741,0.9834,0.9656,0.9783,0.9790]^{T}, \\
{ }_{\mathrm{s}}^{4} \mathbf{u}^{\mathrm{n}}=[0.7713,0.4441,-0.3741,0]^{T}, \\
{ }_{\mathrm{s}}^{4} \mathbf{x}^{\mathrm{n}}=[0.9176,0.9414,0.8355,0.8618,0.9225]^{T}, \\
{ }_{\mathrm{s}}^{3} \mathbf{u}^{\mathrm{n}}=[0.5724,0.4441,-0.7402,-0.1072]^{T}, \\
{ }_{\mathrm{s}}^{3} \mathbf{x}^{\mathrm{n}}=[0.8449,0.8991,0.6724,0.7125,0.8507]^{T}, \\
{ }_{\mathrm{s}}^{2} \mathbf{x}^{\mathrm{n}}=[0.7231,0.8399,0.4505,0.4787,0.6900]^{T}, \\
{ }_{\mathrm{s}} \mathbf{u}^{\mathrm{n}}=[0.1864,0.4441,-1,-0.4019]^{T}, \\
{ }_{\mathrm{s}} \mathbf{x}^{\mathrm{n}}=[0.6202,0.7929,0.3348,0.3788,0.6114]^{T} .
\end{gathered}
$$

The piecewise-continuous linear differential and linear algebraic equations systems matrices are estimated through (9). There are $k=1, \ldots, 2001, \Delta t=0.025$ and the weight matrices are identity matrices.

$$
\begin{aligned}
{ }^{6} \mathbf{A}= & {\left[\begin{array}{ccccc}
-1.8208 & 1.1770 & 0 & 0 & 0 \\
0.0697 & -1.6507 & 0 & 0 & 0 \\
9.7385 & 11.5191 & -20.1171 & -13.1868 & 0 \\
9.6711 & 3.5115 & -5.5917 & -19.3568 & 0 \\
2.9867 & -6.8163 & -3.4159 & -3.1171 & -30.0422
\end{array}\right], } \\
{ }^{6} \mathbf{S}= & {\left[\begin{array}{ccccc}
0.4432 & 0.6940 & -0.0939 & 0.0122 \\
0.2935 & 0.3768 & -0.0274 & -0.1330 \\
0.6187 & -0.5341 & 0.0374 & 0.0125 \\
0.5023 & -1.4456 & 0.0484 & 0.0045 \\
0.4111 & 0.3198 & -0.0392 & -0.0115
\end{array}\right], } \\
{ }^{5} \mathbf{A}= & {\left[\begin{array}{ccccc}
-2.0989 & 1.0320 & 0 & 0 & 0 \\
0.1873 & -1.8560 & 0 & 0 & 0 \\
9.7914 & 11.5725 & -20.0301 & -13.1503 & 0 \\
9.7835 & 3.6756 & -5.5720 & -19.2893 & 0 \\
2.8621 & -6.9600 & -3.4286 & -3.2235 & -29.9508
\end{array}\right], }
\end{aligned}
$$




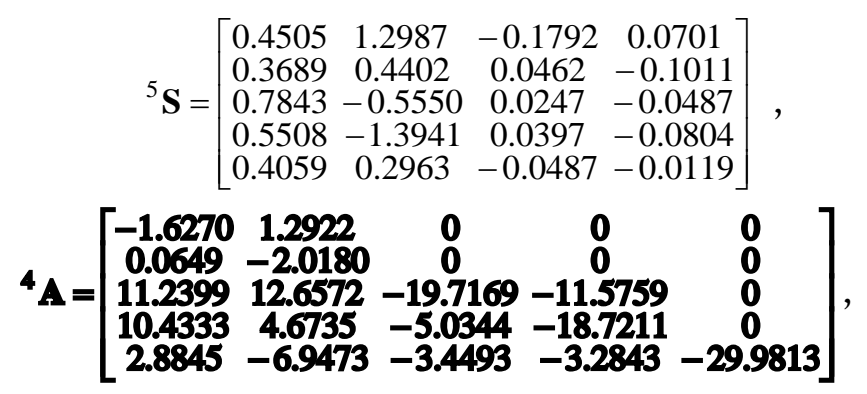

$$
\begin{aligned}
& { }^{4} \mathbf{S}=\left[\begin{array}{cccc}
0.5566 & 0.8041 & -0.0313 & -0.0011 \\
0.3099 & 0.2958 & -0.0303 & -0.1396 \\
0.7465 & -0.3037 & 0.0116 & 0.0521 \\
0.7995 & -1.1151 & 0.0650 & 0.0749 \\
0.3654 & 0.0366 & 0.0288 & 0.0951
\end{array}\right], \\
& { }^{3} \mathbf{A}=\left[\begin{array}{ccccc}
-2.3519 & 1.7300 & 0 & 0 & 0 \\
0.1079 & -2.8400 & 0 & 0 & 0 \\
13.2768 & 14.6302 & -18.6061 & -10.3892 & 0 \\
9.7373 & 4.2626 & -4.2626 & -15.6408 & 0 \\
2.8276 & -3.5453 & -3.5453 & -3.2605 & -30.0025
\end{array}\right] \text {, } \\
& { }^{3} \mathbf{S}=\left[\begin{array}{cccc}
0.4548 & 0.7828 & -0.1086 & -0.0326 \\
0.2726 & -0.0055 & -0.0727 & -0.1164 \\
0.8365 & -0.1525 & -0.0820 & -0.0375 \\
0.7451 & -0.7098 & -0.0892 & 0.0354 \\
0.6537 & -0.1302 & -0.0080 & -0.0835
\end{array}\right], \\
& { }^{2} \mathbf{A}=\left[\begin{array}{ccccc}
-1.7279 & 1.9401 & 0 & 0 & 0 \\
0.5693 & -2.5114 & 0 & 0 & 0 \\
12.8263 & 14.9954 & -18.7520 & -11.9884 & 0 \\
11.4961 & 6.4707 & -4.5819 & -17.7373 & 0 \\
2.8544 & -6.8024 & -3.4658 & -3.2515 & -29.9919
\end{array}\right], \\
& { }^{2} \mathbf{S}=\left[\begin{array}{cccc}
1.0688 & 0.3420 & 0.0642 & -0.0044 \\
0.4710 & 0.0702 & -0.0412 & -0.0549 \\
1.1065 & 0.0011 & -0.0034 & 0.0544 \\
0.9575 & -0.4338 & 0.0132 & 0.0222 \\
0.8451 & -0.0540 & 0.0837 & -0.0729
\end{array}\right], \\
& { }^{1} \mathbf{A}=\left[\begin{array}{ccccc}
-1.9944 & 0.9354 & 0 & 0 & 0 \\
1.2707 & -2.6249 & 0 & 0 & 0 \\
12.5384 & 14.7670 & -18.6298 & -11.9759 & 0 \\
11.4520 & 6.6307 & -4.5503 & -17.6851 & 0 \\
2.0775 & -7.2209 & -4.9486 & -3.4682 & -29.4721
\end{array}\right] \text {, } \\
& { }^{1} \mathbf{S}=\left[\begin{array}{cccc}
0.8329 & 0.5915 & -0.0800 & -0.0486 \\
0.9482 & 0.0695 & 0.0659 & -0.0259 \\
1.4268 & 0.0826 & -0.0835 & 0.0146 \\
1.0210 & -0.3162 & -0.0515 & 0.0026 \\
0.7896 & -0.0358 & -0.0073 & 0.0527
\end{array}\right]
\end{aligned}
$$

The comparison of nonlinear and piecewise continuous differential equations systems state variable $x_{5}^{\mathrm{n}}$ time responses are presented at Figures 1, 2. The errors of piecewise continuous approximation are quite acceptable. 


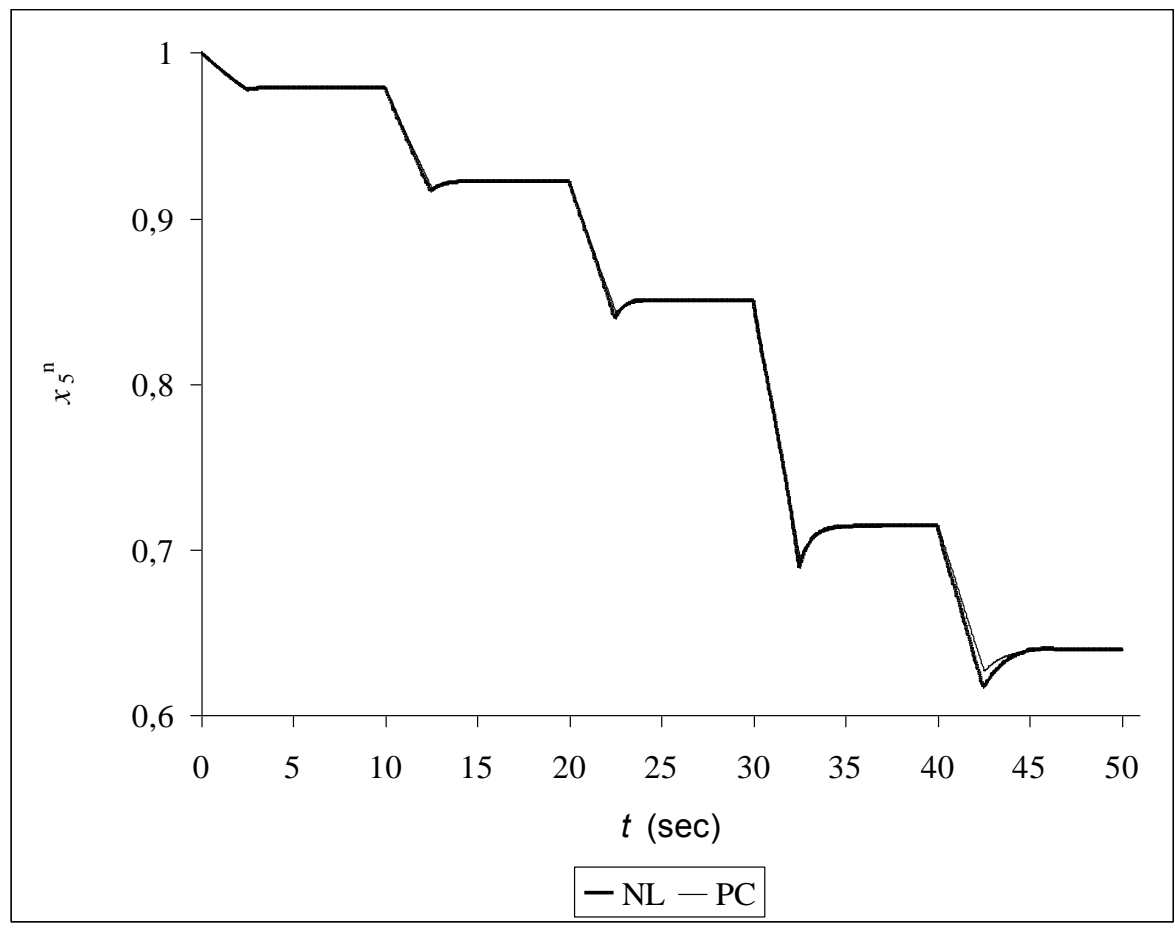

Fig. 1. Nonlinear differential equations system variable $x_{5}^{\mathrm{n}}$ time response that was used for piecewise-continuous differential and algebraic equations systems matrices estimation and corresponding piecewise continuous differential equations system variable $x_{5}^{\mathrm{n}}$ time response.

The errors of the state variables derivatives time responses are most significant. The piecewise continuous, piecewise linear and improved piecewise linear differential equations systems state variable $x_{5}^{\mathrm{n}}$ derivative time responses are presented at Figures 3, 4. These improved piecewise linear differential equations system matrices, piecewise linear algebraic equations system matrices and vectors of normalized input and state variables are calculated through piecewise continuous differential equations system matrices, piecewise continuous algebraic equations system matrices and vectors of normalized input and state variables using equations (6), (7), (8) every $0.1 \mathrm{sec}$.

The comparison of piecewise continuous linear differential equations and piecewise linear differential equations systems state variable $x_{5}^{\mathrm{n}}$ derivative time responses are presented at Figure 3. The piecewise linear differential equations system state variable $x_{5}^{\mathrm{n}}$ derivative time response errors are very large.

The comparison of piecewise continuous linear differential equations and improved piecewise linear differential equations systems state variable $x_{5}^{\mathrm{n}}$ derivative time responses are presented at Figure 4.The improved piecewise linear differential equations system state variable $x_{5}^{\mathrm{n}}$ derivative time response errors are much smaller than those of piecewise linear approximation because an increase in the number of separate linear differential equations systems areas leads to a reduction in errors at the boundaries of these areas. 


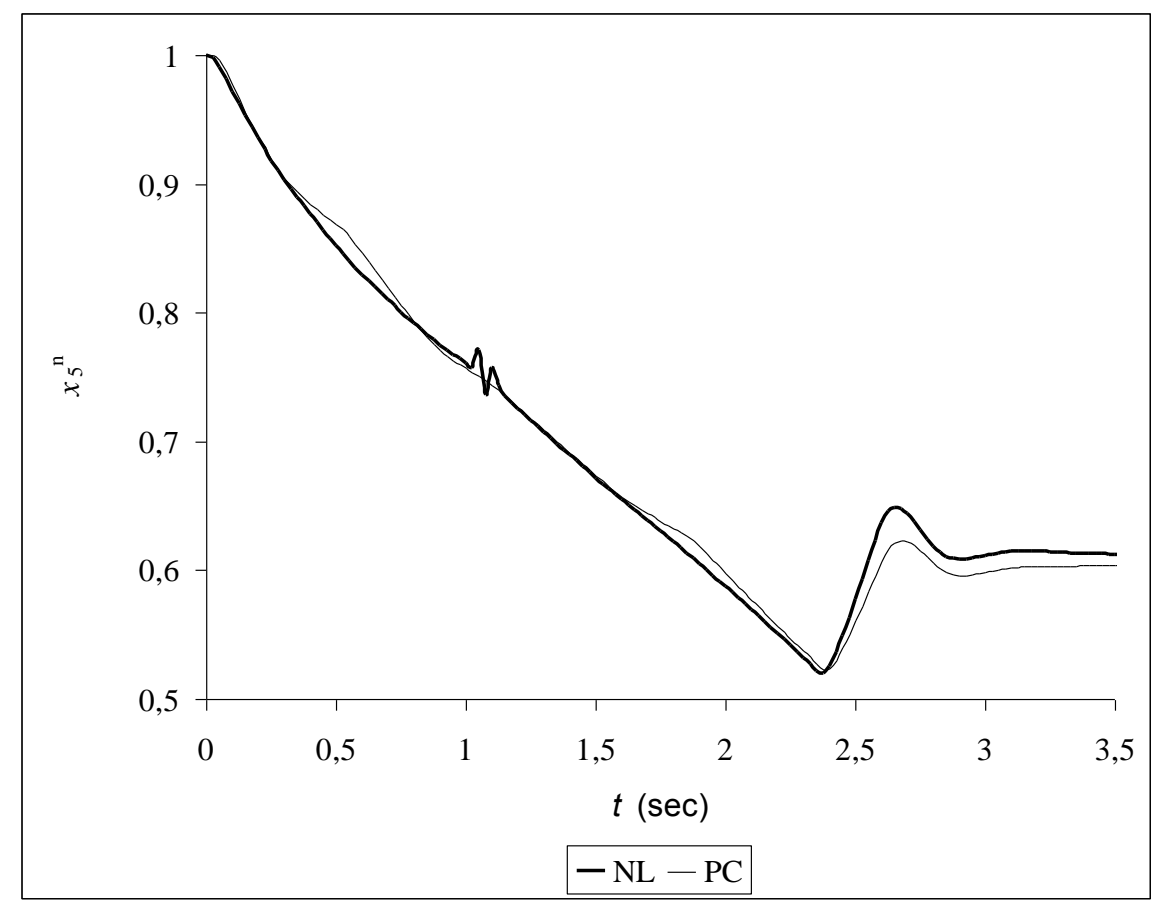

Fig. 2. Comparison of nonlinear differential equations system and piecewise continuous differential equations system variable $x_{5}^{\mathrm{n}}$ time responses.

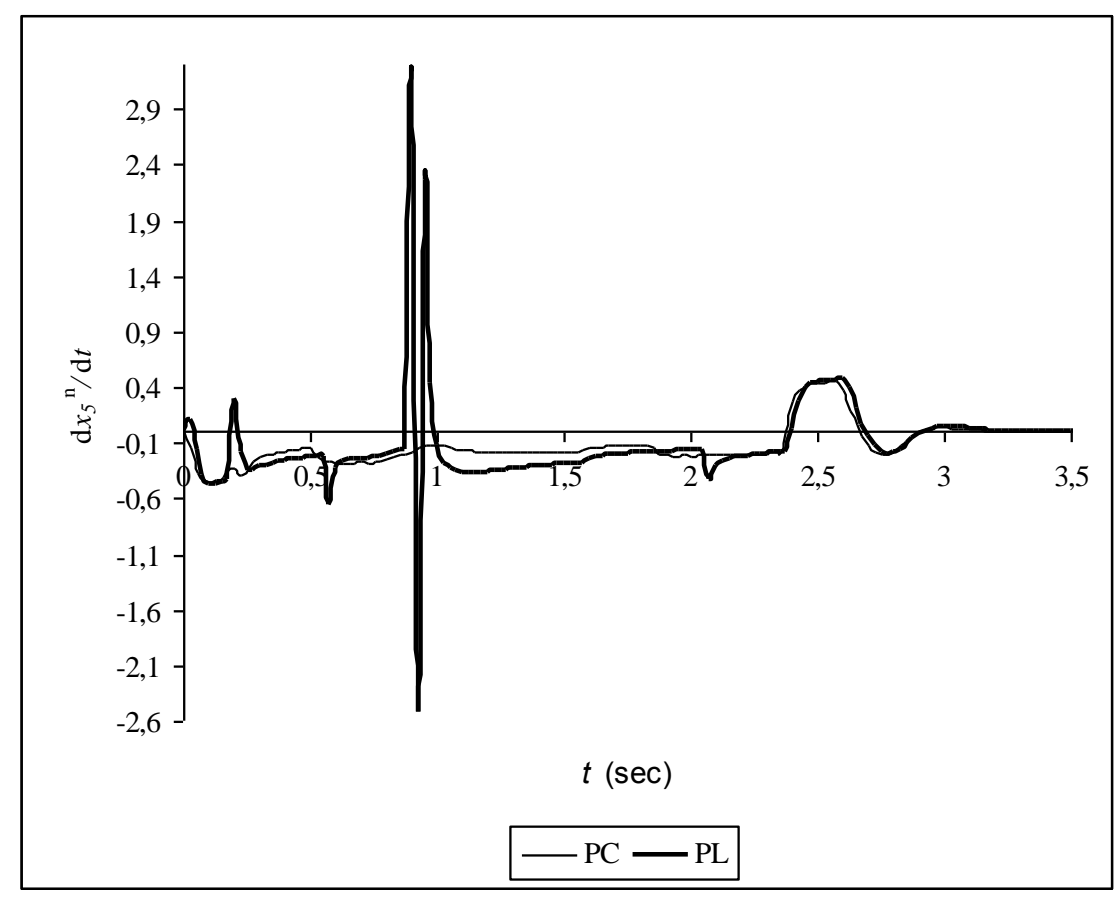

Fig. 3. Comparison of piecewise continuous differential equations system and piecewise linear differential equations system variable $x_{5}^{\mathrm{n}}$ derivative time responses. 


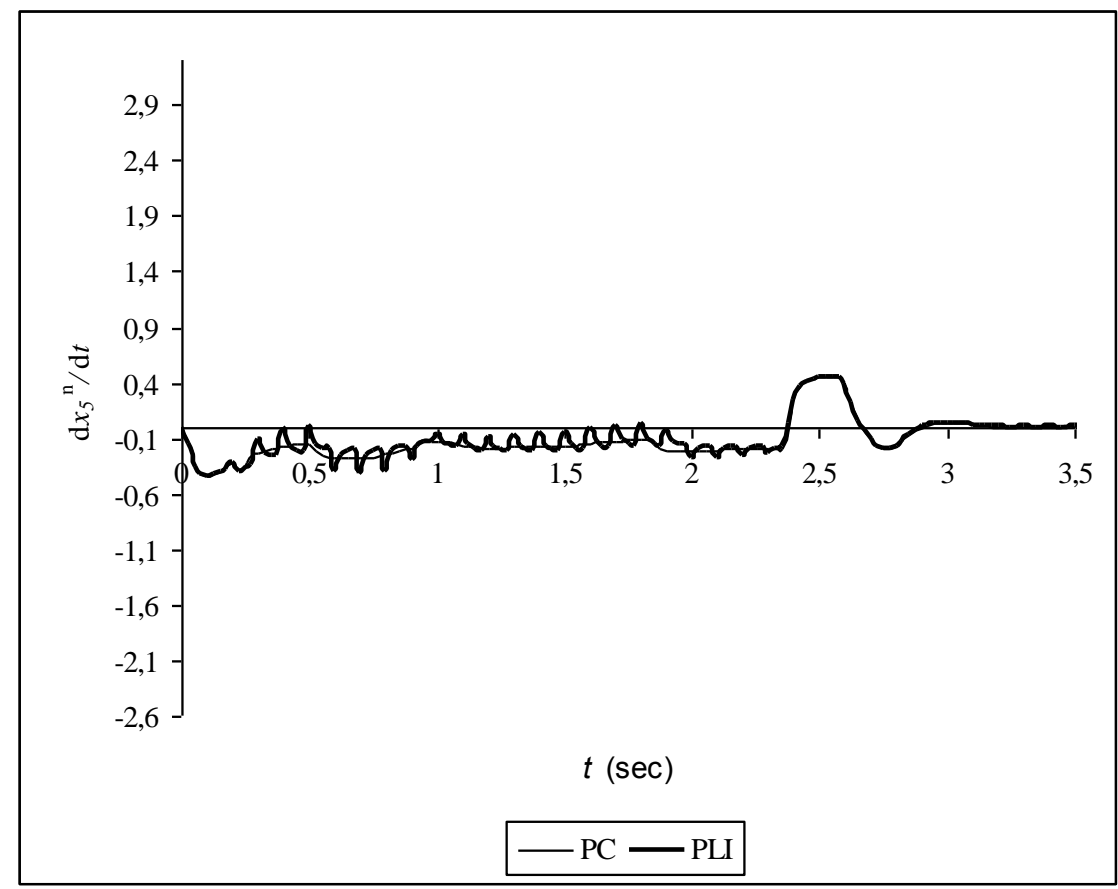

Fig. 4. Comparison of piecewise continuous differential equations system and improved piecewise linear differential equations system variable $x_{5}^{\mathrm{n}}$ derivative time responses.

\section{References}

1. R.L. Leibov, IJCCSE 8, 1, 96-107 (2012)

2. R.L. Leibov, IJCCSE 9, 4, 186-198 (2013)

3. R.L. Leibov, IJCCSE 12, 3, 40-57 (2016)

4. R.L. Leibov, IJCCSE 13, 3, 77-85 (2017) 